\title{
LA TASA DE CRECIMIENTO ECONÓMICO COMO LA VERDADERA CAMISA DE FUERZA DE LA DOLARIZACIÓN
}

ECONOMIC GROWTH AS DOLLARIZATION'S TRUE STRAITJACKET

\author{
Emilio José Calle,Msc. ${ }^{1}$, Fernando Calle W, ${ }^{2}$ \\ TFundación para la Productividad, la Transformación y el Desarrollo del Ecuador (PROTRADE) \\ Universidad Tecnológica ECOTEC Vía Samborondón Km. 13.5 \\ 2 Universidad Católica de Santiago de Guayaquil. Fernando.calle@cu.ucsg.edu.ec
}

RESUMEN

ABSTRACT

La evidencia empírica de la Dolarización ha probado falsa la promesa de que la inflación de los países dolarizados y la del país ancla (Estados Unidos) iban a converger; dejado abierto el debate de que, con un cambio super-fijo como es el de la Dolarización, cuál o cuales son las variables que también se restringen para compensar la rigidez de la moneda dura? Aquí se propone y se presentan soportes teóricos sobre el por qué es el crecimiento económico esa variable que se limita y se confina con la aplicación de un régimen de Dolarización. Esto se logra aplicando una modificación muy particular del modelo del crecimiento económico de Sollow bajo el mercado tecnológico que propone Romer, para ajustarlo a la situación específica del país dolarizado; lo que permite alcanzar la conclusión de que la dolarización impone simultáneamente un piso y un techo al crecimiento económico de todo país viviendo con tal medida.

PALABRAS CLAVE: Dolarización, Crecimiento Económico, Tecnología, Endógeno, Exógeno, Romer Sollow

The empiric evidence from Dollarization has proven false the premise that the inflation of the dollarized nations and the one from the anchor country (the US) will converge; opening up the debate about which variable or variables a super-fix exchange system such as Dollarization is restricting to compensate for this lack of flexibility? The proposal here, supported over formal economic theory, is that it's economic growth the variable that is modified and confined under a dollarization regime. This is accomplished through the application of a modified Sollow economic growth model with Romer's technology market concepts, however tweaked to the particular circumstances of a dollarized economy. The beforementioned model concludes that dollarization imposes simultaneously a Roof and $a$ Floor on the economic growth rate that a dollarized economy can achieve.

KEYWORDS: : Dollarization, Economic Growth, Technology, Endogenous, Exogenous, Romer Sollow 


\section{Introducción}

En trabajos anteriores se ha demostrado que la Dolarización no es la eliminación de la política cambiaria de un país sino el reemplazo de un tipo de cambio variable a uno super-fijo (Nudelsman, 2017). Y también se demostró que cambiar una variable (tipo de cambio variable), a una constante (cambio fijo) en la función de producción de un país endosa más peso al resto de variables, restringiendo y anclando los límites que pueden alcanzar el resto de variables. (Calle, 2019)

Tradicionalmente se consideró que era la inflación la que iba a ser la "camisa de fuerza" de la Dolarización, es decir, que iba a ser igual la inflación del país dolarizado que la del país ancla (Sachs \& Larrain, 1999); esto no se ha observado en la realidad de los resultados de los países dolarizados actualmente (El Salvador, Ecuador y Panamá). Pese a que las tasas de inflación han bajado, en ningún caso esas inflaciones son iguales a las de Estados Unidos, lo cual se demostró usando análisis econométricos que corroboraron que ni la inflación americana ni sus componentes servían de base para pronosticar las inflaciones de los otros países dolarizados y viceversa (Calle, 2018); lo cual implicaba que no se podían considerar iguales a estas dos variables.

Aquí planteamos el tema desde otra perspectiva: si no es la inflación, ¿cuál es la camisa de fuerza que se ocasiona a raíz de implementar un régimen de Dolarización? En este documento se construye sobre la base del cambio de moneda super fijo, que mantiene los sueldos reales elevados, y a su vez las rentas elevadas, para explicar el por qué la Dolarización "corta" al crecimiento económico en su parte de arriba (la innovación del país Dolarizado, manga Alta de la Dolarización); y en su parte de "abajo" (atraer a industrias de producción barata, transitivas); haciendo de esta manera que, "de facto", la Dolarización ancle el crecimiento económico del país dolarizado (Castillo \& Rodriguez, 2009); siendo el propósito de este ensayo comprobar si coincide con el del "'país ancla”, esto es, el país soberano de la moneda.

Gregory Mankiw dice que el propósito del análisis macroeconómico es el de explicar por qué crece el ingreso de una nación, y por qué los ingresos de una nación crecen más rápido que el de las otras. (Mankiw, 2007) Por dicho motivo, cabe profundizar en los dos modelos de crecimiento a considerar en el presente estudio: el modelo de Sollow y el de Romer. El modelo de Robert Sollow, ganador del premio Nobel de economía en 1987 (Policonomics, 2017), nace como una respuesta al modelo de crecimiento económico de Harrod-Domar, el cual presentaba al crecimiento económico como una función basada en la tasa de ahorros y el retorno a la inversión. (Mixon, 2007) Sollow descubrió que este modelo era irreal puesto que dependía mucho de que las escalas económicas sean constantes, es decir, que el ratio de mano de obra y capital para la elaboración de cualquier producción se mantuviera estable en el tiempo, lo cual es muy poco probable dado que el progreso tecnológico y los cambios demográficos hacen que estos dos factores aumenten (o disminuyan) sus productividades de maneras diferentes. (Sollow, 1994) Modificando el modelo de Harrod-Domar para contabilizar estas diferencias, el modelo de Solow permite incluir un elemento de tecnología, llamado la Productividad Total de los Factores (z), que permite "endogenizar" el crecimiento económico (BAROSSI-FILHO, et al., 2004) Construyendo sobre lo desarrollado por Solow, Romer se concentró en cambio en analizar el "mercado" para el desarrollo de la tecnología, de dónde venían estas innovaciones que aumentaban tanto la productividad y generaban el crecimiento. (Jones, 1995) También ganador del Nobel por este trabajo (Redacción $B B C, 2018$ ), Romer se enfocó en el hecho de que la evidencia empírica muestra que el modelo de Solow calza mejor con la evidencia de los resultados de las naciones cuando no solo aumenta el capital físico sino el humano también, mediante educación entre otras variables. Este capital humano al acumularse facilita la gestación de innovación y nuevas tecnologías que son la fuente del crecimiento económico a largo plazo. (Bernanke \& Gtirkaynak, 2001)

Finalmente se advierte el no dejar de lado un concepto muy significativo para este caso: la diferencia entre el Crecimiento Económico Nominal y el Crecimiento Económico Potencial, basado en el PIB potencial de los países (Thurow \& Taylor, 1966). Este es un tema muy largo, meritorio de su propio artículo, pero que se vuelve relevante de mencionar en la coyuntura de este tema en particular.

\section{Metodología}

La parte más novedosa y lo que más valor agrega a este trabajo es precisamente esta sección pues, hasta donde hemos podido investigar, jamás han sido usadas estas bases teóricas en un contexto de Dolarización, pese a su popularidad al ser parte de un triunvirato de premios nobeles en Solow, Romer, y en un rasgo más general, las teorías Mundell respecto a las Zonas Monetarias Optimas. Es evidente el por qué: en el mundo desarrollado el tema de la Dolarización se ve muy lejano y descabellado, tanto, que no ha sido necesario explorar la posible adaptación a esa realidad de las teorías aquí presentadas. Pensamos que hoy contribuimos al zanjar esa brecha. 


\section{Materiales y métodos}

El presente trabajo se basa en dos líneas de materiales teóricos: el Modelo de Crecimiento Endógeno de Romer, el cual se utilizará para buscar una explicación del por qué un país dolarizado no puede generar crecimiento económico autónomo a través de la innovación; y el modelo de competencias absolutas y comparativas de David Ricardo, (Cely \& Calle, 2019) el cual se utilizará como base teórica para explicar por qué un país dolarizado no puede generar crecimiento económico atrayendo la re-ubicación de industrias de otros países al territorio nacional (industrias transitivas).

El modelo de crecimiento endógeno de Romer, recientemente galardonado con el premio Nobel de Economía, existe desde 1986 y se creó como respuesta al modelo de convergencia económica de Solow (mostrado más adelante en este trabajo), el cual muestra cómo los países pueden alcanzar un equilibrio macroeconómico en términos de niveles óptimos de todas sus variables, pero que no podía explicar el por qué las economías podían seguir creciendo más allá de este equilibrio; es más, pronosticaba que una vez alcanzado este punto óptimo las economías dejaría de crecer, lo cual no era compatible con la evidencia arrogada por las cifras reales. (Whelan, 2014)

Se empleará también el concepto de retornos de escala, para definir los diferentes tipos de industrias en economía internacional, clasificándolas como Transitivas, No Transitivas, y Clústers, según su comportamiento en escalas económicas. Finalmente se tocará en el modelo de Sollow respecto a la convergencia económica a largo plazo. (Williams, 2017)x`

El retorno marginal al trabajo ( $\mathrm{mrl})$, la inversión y la tecnología

Construyendo sobre lo expresado anteriormente, se empieza a construir el modelo de la camisa específica del Ecuador, en este caso de la "manga superior" de la misma. Esta parte explicará por qué el Ecuador está limitado en sus posibilidades de crecimiento económico debido a la Dolarización, siendo este el límite alto de la camisa.

Esta segunda parte explica por qué en el Ecuador la Dolarización facilita y fomenta la adquisición e implementación de la tecnología creada en otras partes debido a la forma en que fortalece al sistema financiero y permite la planeación al largo plazo. Se demostrará que la implicación de que el sector financiero ecuatoriano esté "protegido" o desconectado de la situación política del país es que, independientemente de cuánto error cometa la sociedad ecuatoriana, habrá siempre un mínimo crecimiento de la economía nacional casi por osmosis a efecto de la Dolarización. Proceso de gestión de datos

Los datos estadísticos aquí presentados provienen de dos fuentes. La primera, respecto a los datos nominales de resultados económicos del Ecuador, estos provienen de distintas fuentes que son: los boletines económicos del Banco Central del Ecuador como fuente principal; los reportes del Banco Federal de Reserva de Estados Unidos (FRED) como respaldo y para corroborar o para complementar datos; y otras fuentes varias que tienen cifras específicas diferentes que sean de utilidad, como por ejemplo los Ministerios de Finanzas y de Economía del Ecuador, la Superintendencia de Bancos, el Fondo Monetario Internacional, o el Banco Mundial.

Estos datos han sido ordenados por fechas y rubros, además de haber sido puestos en términos reales (sin inflación), y en la misma moneda cuando correspondía. Finalmente se ha recurrido a varios libros y reportes históricos para analizar datos correspondientes a periodos pre-dolarización e incluso pre-democracia (1978, cuando termino la dictadura militar).

\section{Procesamiento de datos}

Debido a que el presente ensayo apunta más hacia proponer y sustentar una teoría respecto al papel que juega el crecimiento económico en un país dolarizado, el procesamiento de datos empleado no requiere herramientas econométricas avanzadas, por lo tanto no se han aplicado técnicas de normalización de datos para eliminar ruidos como la estacionalidad, o la heteroscedasticidad.

\section{Resultados}

Esta sección capitaliza sobre teorías económicas formales para explicar los dos grandes efectos que tiene el usar una moneda de cambio fijo (dólar) sobre el crecimiento económico de un país dolarizado como el Ecuador. El primer efecto que se demuestra es que le pone un TECHO teórico al crecimiento económico, el cual nunca podrá ser superior al crecimiento económico del país ancla (donante de la moneda); debido al efecto que tiene la Dolarización sobre el valor real de los salarios en la economía dolarizada. Por otro lado, la Dolarización también presta un PISO, o valor Mínimo de crecimiento económico que se debiera tener, debido a que con tasas de interés más bajas y con mayor estabilidad financiera, se pueden implementar más proyectos con puntos de equilibrio más distantes en el futuro que, por ejemplo, en un país subdesarrollado no dolarizado. El argumento final reza que será entre estos dos límites teóricos, se ubicará el crecimiento económico real de todo país Dolarizado. 


\section{El modelo de crecimiento económico de Romer}

La innovación del modelo de Romer fue el introducir el concepto de la existencia de un mercado del conocimiento, el cual generaba aumentos de la productividad (Factores Totales de Producción - TFP), resultando en un equilibrio de crecimiento positivo y creciente basado en la endógena acumulación de conocimiento. Este fue un importante distanciamiento de la literatura existente hasta ese momento puesto que se había considerado al avance tecnológico como una variable exógena, es decir, que ocurría casi por suerte o al azar (Carroll, 2017).

Debajo se presenta un gráfico que muestra como las teorías de Romer logran causar el efecto descrito arriba:

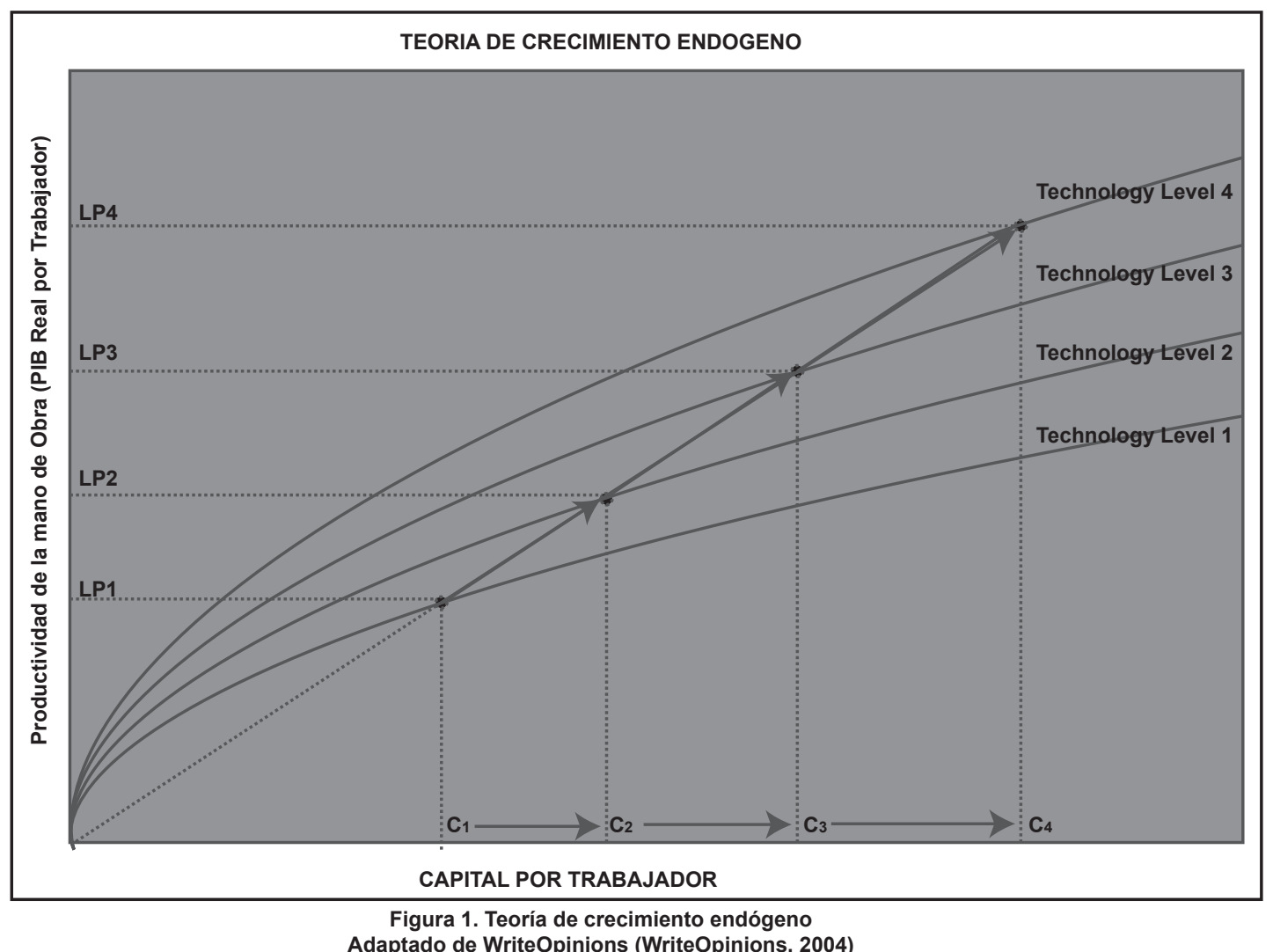

Como se puede observar, el incremento tecnológico (TFP) logra aumentar la capacidad de producción de una economía, dado que la tecnología vuelve más productivos tanto al capital (K) como al trabajo (L), según lo mostrado en la fórmula:

$$
\mathrm{Y}=\mathrm{A}(\mathrm{K}, \mathrm{L})
$$

Considerando que "A" afecta a ambos lados de la ecuación de la producción, el aumento tecnológico levanta a todas las variables de la economía al mismo tiempo, con lo cual crea un nuevo equilibrio más grande, de mayor riqueza, lo cual se puede observar en el aumento de capital por trabajador (lo cual es lo mismo que la cantidad de capital por persona en la economía dado que $\mathrm{L}=$ $\mathrm{n}$, y más capital es igual a mayor riqueza).

\section{Definición de países desarrollados y subdesarrollados}

Combinando los conceptos anteriormente tratados respecto a lo que es Desarrollo
Económico, y de crecimiento mediante innovación tecnológica, surge una definición que se utilizará de forma exclusiva para este análisis dado que no es un canon económico aceptado: el de lo que es un país desarrollado versus lo que es un país subdesarrollado.

Muy específicamente se utilizará esta definición para referirse a Estados Unidos y Ecuador. Evidentemente, Estados Unidos es el país desarrollado, pero la pregunta es por qué. Usando los conceptos anteriores, la respuesta es que Estados Unidos es un país desarrollado puesto que ha alcanzado un nivel en el cual sus instituciones y su nivel de régimen de justicia no sólo protegen, sino que promueven la innovación tecnológica y el crecimiento económico a través de esta. La prueba está en el número de patentes otorgadas por año: según los Indicadores de Propiedad Intelectual de la ONU, Estados Unidos logró más de 300,000 aprobaciones de patentes en el 2016, segundo sólo a China, que fue primera con un poco más de 400.000 (WIPO, 2017). 
Por otra parte, Ecuador es un país totalmente carente de desarrollo tecnológico, demostrado en el mismo reporte antes mencionado donde no aparece ni siquiera con una aplicación, menos aún con una patente otorgada. Esto se debe, en parte, a la nula institucionalidad del Ecuador y a un sistema legal débil que desestimula cualquier inversión o intento de desarrollo intelectual. En ningún lugar es esto más prevalente que en la "bahía" de Cuayaquil, un mercado informal que figura entre los cinco más grandes del mundo en lo que a piratería se refiere (El Universo, 2011)

Para resumir, se usa el concepto de desarrollo de tecnología de Romer para definir a Estados Unidos como un país desarrollado por su amplia creación tecnológica, mientras que se define a Ecuador como un país subdesarrollado que carece totalmente de esta creación, aunque cabe recalcar que esto no significa que no tenga tecnología, sino que significa que la tiene que adquirir fuera de sus fronteras. Esta última aclaración será muy importante para las secciones posteriores

\section{Determinación de los costos del desarrollo de la tecnología}

El siguiente concepto importante de entender es que hay un costo inherente en desarrollar tecnología. Este costo comprende los salarios de los investigadores (generalmente profesionales y especialistas de sus áreas, con salarios más altos que el del trabajador promedio); costos de implementos, instalaciones, años de estudios, otras patentes ya desarrolladas, etc. (Robb, 2018). Por ende, Romer explica que habrá instancias donde la tecnología desarrollada se implemente, y habrá otras en las que no, lo cual dependerá del retorno a escala que la tecnología genere. Así, si la tecnología genera retornos a escala crecientes o constantes, será rentable implementar la tecnología. Caso contrario, si el retorno a escala es decreciente, entonces será mejor no implementar la tecnología

Este último caso implica un costo hundido adicional para el desarrollo tecnológico: si se emplean recursos en intentar desarrollar una tecnología que no se implementa, al final se pierden estos recursos y la sociedad tendrá que reponerlos aumentándolos al costo de cualquier otra tecnología que sea exitosa en el futuro.

El siguiente es un ejemplo de cómo Romer plantea la forma en que se efectúan los costos del desarrollo de una tecnología:

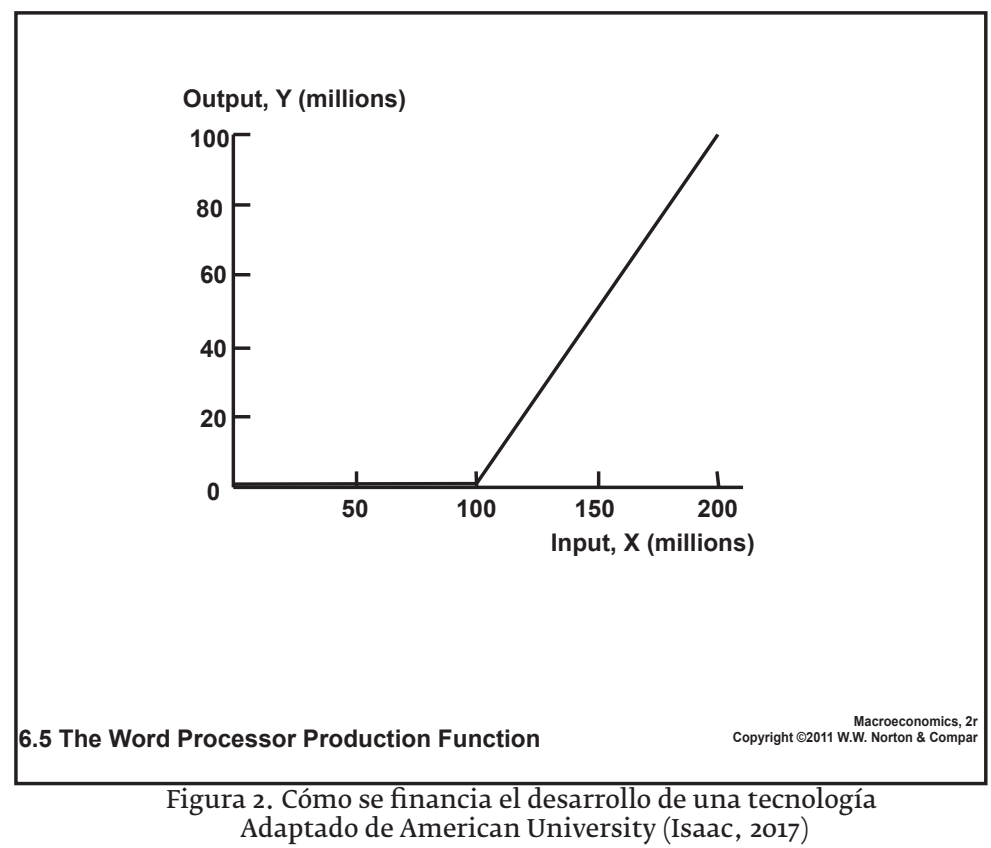

Como se puede apreciar en este ejemplo, el desarrollo de esta tecnología (un procesador de palabras), tiene un desembolso inicial de \$100 millones antes de empezar a generar ingresos. El punto de Romer es que, si este costo inicial no es cubierto, la tecnología no se implementará y lo que se haya gastado en su desarrollo será tan sólo un costo hundido. Esta recuperación sólo sucederá si la tecnología es aplicada a

industrias donde pueda tener retornos a escala constantes o crecientes. Si fueran decrecientes, la curva no giraría para arriba sino para abajo, haciendo inútil el esfuerzo del desarrollo de la tecnología. Cabe recordar lo que significa el concepto de retorno de escala - es cuanto producto adicional se genera por multiplicar todos los factores por un vector X. Si el resultado es que el nuevo producto es igual a $\mathrm{YX}=1$, 
entonces los retornos a escala son constantes: cualquier cambio proporcional de los insumos genera un cambio de producción equiproporcional. Si YX>1, entonces los retornos a escala están aumentando, es decir que 1 dólar de inversión en insumos genera más de \$1 en producto final; lo contrario ocurre con retornos a escala decreciente. (Diewert, Nakajima, Nakamura, Nakamura, \& Nakamura, 2011)

\section{Clasificando las industrias en transitivas y no transitivas}

Nuevamente hay que crear una definición basada en dos conceptos presentados anteriormente: los costos de tecnología, y los retornos a escala. A estos se les sumará la posibilidad de comercio entre los países, específicamente la transferencia tecnológica entre estos; lo cual normalmente sería cubierto o por economía internacional o por finanzas internacionales, pero que sin embargo se tratará de manera tangencial aquí.

Se expuso anteriormente que, si la implementación de la tecnología no lograba cubrir sus costos de su desarrollo, entonces esta no se ejecutará. Sin embargo, es totalmente posible que los costos de implementación de una tecnología varíen de un país a otro, debido al nivel de desarrollo que puedan tener en el momento que salen al mercado. Así, una tecnología que no sea rentable en un país como Estados Unidos, podría ser rentable en otro país donde los costos de implementación sean bajos, siempre y cuando dicho país tenga acceso a mercados igual o más grandes que el país originario de la tecnología-es decir, es un bien TRANSABLE, un bien que puede ser producido en otro país que el que se consume, sin pérdida de calidad o volumen (y generalmente de forma más barata). Un caso de este tipo podría ser México, un país que evidentemente está lejos de alcanzar su tasa de crecimiento económico de equilibrio pero que sin embargo tiene amplio acceso al mercado americano. Si este es un caso donde la tecnología puede ser transferida para que sea rentable, entonces esta es una industria transitiva, es decir, una industria que puede libremente ejercerse afuera de las fronteras del país inicial sin afectar el producto o servicio final. Gráficamente se podría representar así:

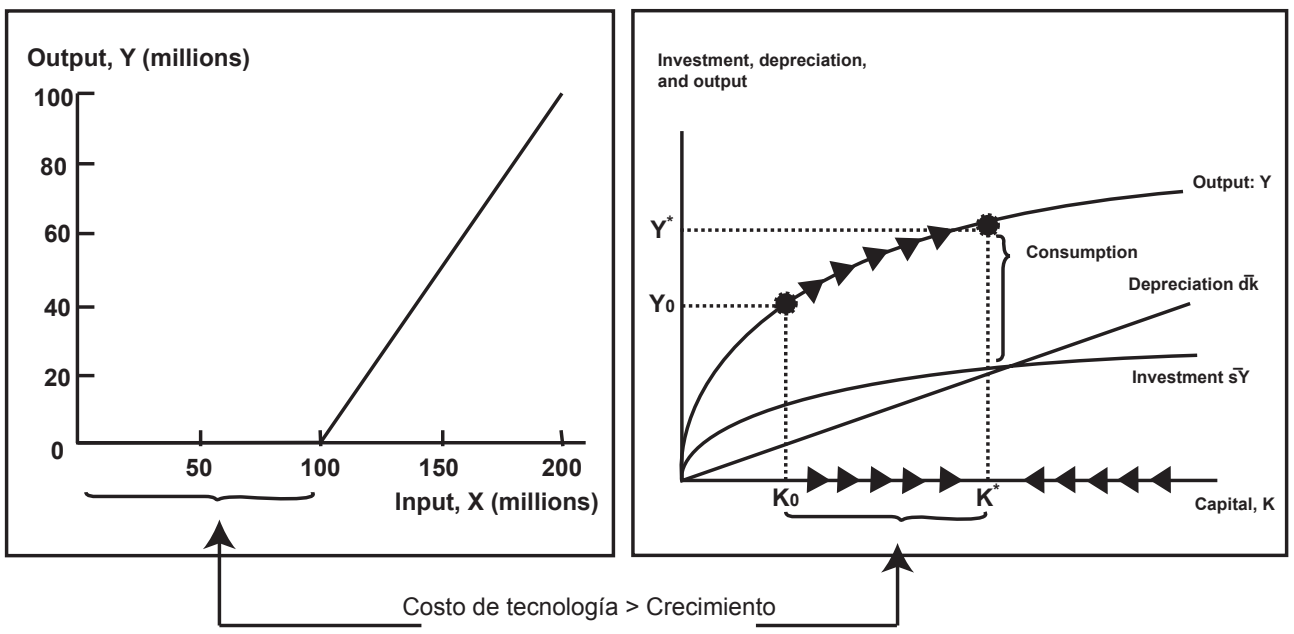

Figura 3. Costo de desarrollo tecnológico en una economía en equilibrio Adaptado de American University (Isaac, 2017).

En el caso que muestra arriba en el Gráfico 3, la tecnología no sería implementada puesto que los costos fijos de desarrollarla son mayores al crecimiento económico esperado, medido en este caso como producción por el capital. Esto sucede porque en el ejemplo dado arriba, la economía comienza ya con su capital en equilibrio (steady state), como se puede ver en las líneas diagonales sobre Yo. (la cantidad de $\mathrm{K}$ es casi paralela a la de depreciación $\mathrm{dK}$, lo que significa que están en equilibrio, es decir, cada capital que se agrega es para reemplazar capital usado o depreciado). Aquí, el costo de la tecnología es 100 millones, mientras que el crecimiento económico es de una magnitud menor, lo que quiere decir que la cantidad de K (Capital) necesario para poder justificar el costo de la tecnología no se da en esta economía, debido a que, como está desarrollada y empieza en equilibrio, el movimiento de $\mathrm{K}$ no es tan grande.

Debajo se muestra el caso opuesto: una economía en la cual el capital no comienza en su estado de equilibrio, brindando campo de crecimiento para que el impacto de la inversión en tecnología no sólo genere el crecimiento económico arriba presentado, sino que lo haga de una manera tal que rinda el valor de la inversión en la tecnología e incluso más. 


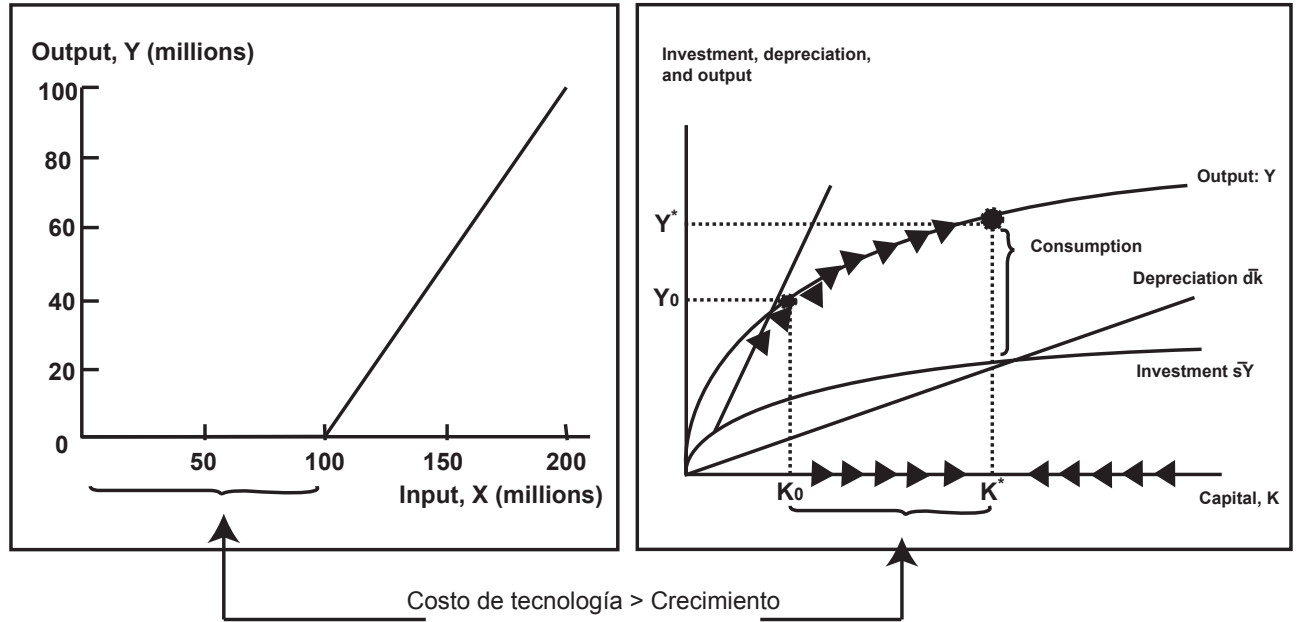

Figura 4 Costo de desarrollo tecnológico en una economía que empieza fuera de equilibrio Adaptado de American University (Isaac, 2017)

En este caso se muestra una economía que comienza fuera de su equilibrio de capital, una economía en desarrollo, donde hay una oportunidad de hacer rentable la innovación tecnológica debido a que tiene más espacio para crecer en capital que una economía desarrollada. Si este caso se da en una industria que pueda ser exportada del país generador de la tecnología, entonces sería lo que se denominaría una economía transitiva. En Estados Unidos esto es exactamente lo que ha sucedido, siendo un caso muy famoso el de los Call Centers transferidos a la India, utilizando la gigantesca caída en los costos de comunicación que conlleva el usar teléfonos IP, esto es, basados en la internet: actualmente más de 265.000 personas trabajan en esta industria en India, y generan casi \$30.000 millones de dólares de ingresos anuales, algo que no pasaría ni en USA ni en Europa dado que este tipo de trabajo es menospreciado por los locales (Hartley \& Walker, 2012).

Para recapitular, las posibilidades de la innovación tecnológica son: ser rentable en el país creado ya sea por tener retornos de escala constantes o crecientes; ser rentable en otro país fuera del originario, con lo cual se la consideraría una tecnología para una industria transitiva; o no ser rentable en ningún caso por lo cual quedaría como costo hundido. Al mismo tiempo esta recapitulación abre el abanico para entender tres clases de industria que pueden darse si la implementación de la tecnología resulta rentable. La primera es la industria No Transitiva, es decir de bienes No Transables que no pueden ser producidos en otros países (como son la mayoría de servicios); que genera retornos de escala crecientes en el país donde se implementa. Ejemplos de industrias No Transitivas, es decir, industrias que no pueden desconectarse de su país de origen o mercado, son por ejemplo casi todos los servicios que requieren contacto personal: doctores, belleza, restauración (hoteles, restaurantes, turismo, etc), leyes, y demás, como por ejemplo los servicios gubernamentales y públicso. Por esto vemos que este tipo de industria tiene ganancias extraordinarias en los países tecnológicamente avanzados, como por ejemplo con abogados que ganan \$1000 o más por hora, consultores en similar situación, atletas, etc. Si por el contrario la industria tiene retornos constantes a la tecnología, entonces es un cluster, lo cual se define como una industria con una cadena de valor globalizada, que ya ha extraído las ventajas competitivas posibles en todas las partes del planeta donde las ha podido conseguir, y que cualquier crecimiento de sus ingresos será exactamente proporcional a la inversión realizada, lo cual implica que se realizará por el simple hecho de que el rol de los empresarios es estar siempre aumentado sus ingresos. Finalmente, si la tecnología sólo resulta rentable saliendo de su mercado de origen en el área de países desarrollados, entonces será una industria Transitiva como ya se explicó anteriormente. Recapitulando lo mencionado anteriormente, hay tres definiciones derivadas del desarrollo de la tecnología: la primera, que los países desarrollados son los que desarrollan la tecnología; y que los países subdesarrollados son los que NO desarrollan tecnologías. Este último punto es relevante puesto que, si se asume que los países no desarrollados no generan tecnología, entonces, ¿de dónde viene el desarrollo tecnológico del que hablan Romer y Solow? Se propone una respuesta debajo.

El segundo punto derivado de los subcapítulos anteriores es que el desarrollo de la tecnología tiene un costo, y es un costo que debe ser devengado en ganancia para el desarrollador privado, pero también como ganancia general 
para la sociedad en donde se aplique esta tecnología. Este punto también es muy extenso, tiene ramificaciones en el área del mercado para tecnología, mercado laboral, educación (mercado de capital humano), etc. No se tratarán ninguno de estos acá, para propósitos de continuar la explicación se simplificará el ejercicio y se dirá que, en aras de buscar cómo lograr que el desarrollo de la tecnología no se desperdicie al no ser costo-eficiente su implementación, los desarrolladores buscan mercados donde se puedan implementar, esto es, buscan llevarlos a países no desarrollados con mayores MPKs y sin tecnología que compita con ellos.

Esto a su vez conduce a los tres tipos de industrias discutidos: las transitivas, no transitivas, y clústers. Las no transitivas y clústers son mercados potenciales para las tecnologías en cualquier país, pero sólo las transitivas son mercados potenciales para aquellas que no son rentables en países desarrollados.

Lo descrito arriba crea dos lados diferenciados del mismo mercado: el de la venta de la tecnología. Por una parte, el país rico que desarrolla la tecnología recupera su inversión si la vende a una industria No Transitiva de su país. Este es el mejor escenario posible ya que el ser NO transitiva implica una situación de falla de mercado: por alguna razón (legal, cultural, etc) este mercado afectado no puede ser "globalizado" o desprendida su producción de su implementación. Es por esto que este tipo de industria se definió arriba como de Retornos de Escala Incrementales, es decir, \$1 de inversión genera más de \$1 de retorno.

En un caso como el arriba mencionado, ganan todos los jugadores internos: gana el desarrollador de la tecnología, gana el em- presario que la implementa puesto que puede aumentar sus ingresos y su utilidad a la vez, y gana la sociedad puesto que se crean más trabajos. Es importante mencionar que el generador de esta tecnología puede potencialmente venderla a otros países que las implementarán en sus propios países, por lo cual no quiere decir que todo el costo del desarrollo de la misma deba ser asumido por el mercado interno (el país rico que la desarrollo), lo cual representa incluso más ganancia para el país generador ya que le reduce costos.

Un caso un poco diferente pasa con los clústers: al ser cadenas de valor totalmente globales, el desarrollo de tecnología solo puede aumentar las ventas, pero no el porcentaje de utilidad (sólo aumenta su valor nominal). De todas maneras, se implementará puesto que todo empresario prefiere tener más ingresos nominales, aunque su ganancia real (como porcentaje de esos ingresos) sea la misma. Es decir, mientras el costo marginal de la tecnología sea igual al beneficio marginal, la tecnología se implementará, pero la utilidad de la industria no crecerá como en el caso anterior (perderá cualquier ganancia extraordinaria en el largo plazo)k, y la ganancia para la sociedad a través de más trabajos no se dará.

Finalmente, en el caso de las industrias Transitivas, sólo el desarrollador de la tecnología gana en el país de origen, y es más, esa ganancia es cancelada por la pérdida social de trabajos que se van al extranjero, como los mencionados respecto a los call centers, o prácticamente todos los trabajos industriales que se han ido de Estados Unidos a China, India y otros países similares. La tabla debajo resume estas ideas:

Tabla 1. Transferencias tecnológicas y ganancias sociales de países desarrollados a sub-desarrollados

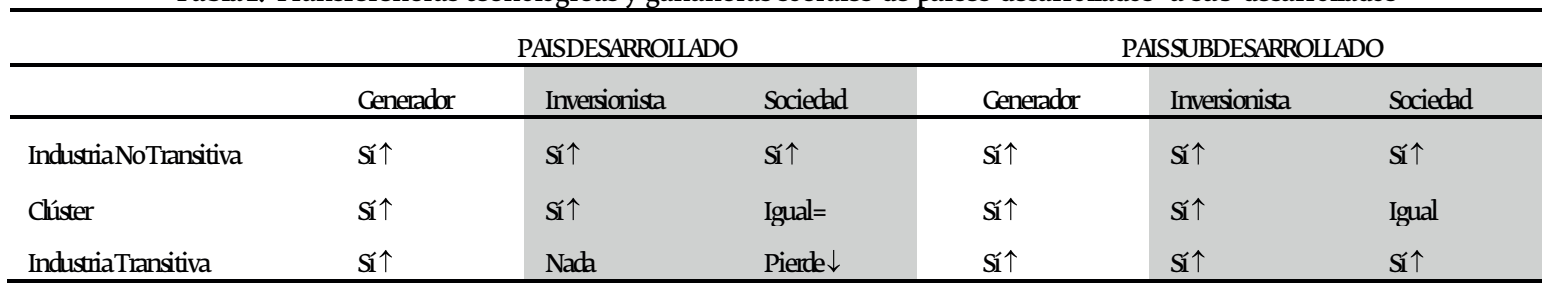

La primera conclusión evidente de la Tabla 3 es que el generador de la tecnología gana en todos los escenarios; esto es evidente porque como se mencionó antes el propósito final era encontrar a un mercado para que la tecnología resultase rentable. Se insiste en que este análisis del mercado global de la tecnología es en extremo simplificado; normalmente este tema se trataría utilizando las herramientas de Finanzas Macroeconómicas Internacionales, pero para el alcance de este estudio la explicación tan grosso modo sigue siendo válida. Tomando como constante que el generador de la tecnología va a encontrar una utilidad en estos mercados, sólo cabe analizar qué resultados obtienen los inversionistas y las sociedades de estos dos tipos de países.

$\mathrm{Y}$ es aquí donde se comienza a notar una gran diferencia: mientras el país desarrollado no gana en 2 escenarios y pierde en un tercero, el país no desarrollado nunca pierde, y solo sale tablas en el caso de los clústers. Es decir, el resultado final para el país desarrollado es un progreso de $2(\uparrow+\uparrow+\uparrow-\downarrow)$ vs una ganancia de 5 para el país no desarrollado $(5 \times \uparrow)$. Evidentemente se repite que este es sólo un ejercicio de lógica, donde se pretende demostrar por qué un país no desarrollado tendría una 
capacidad y aceleración de crecimiento tan o mucho más rápida que un país desarrollado, pero de ninguna manera se quiere establecer que esa relación es en un ratio de 5/2. Es simplemente un ejemplo para demostrar este mecanismo, y debe ser tomado como tal.

En el caso de los países desarrollados, el mayor punto que se quiere hacer notar es que el desarrollo de tecnología per se podría no generar crecimiento: si la industria se va del país, sí, los creadores de la tecnología y potencialmente los dueños de las empresas ganaran dinero (utilidades), pero se perdieran trabajos y se perdieran ingresos sociales como impuestos y las externalidades positivas de la creación de trabajos. Es decir, la generación de tecnología en una industria transitiva termina siendo perjudicial para la sociedad desarrollada, y nulifica el efecto de la generación de esta tecnología aun cuando esta logre beneficios en la forma de utilidades privadas.

En el caso de los países no desarrollados esto no sucede puesto que el gasto en la tecnología es o, es decir, no hay impacto si se genera una tecnología que no encuentre mercado o que no sea costo eficiente, por el simple hecho de que no se generan tecnologías y punto. No hay trabajos que destruir porque no existían para empezar.

Manga Baja de la Camisa: Por qué la Dolarización impide que este mecanismo se aplique en el Ecuador

Utilizando los largos antecedentes presentados en este estudio, se va a ana- lizar específicamente el por qué la Dolarización le impide a Ecuador ser un jugador de la dinámica del acápite anterior, explicando al mismo tiempo por qué se limita el crecimiento económico ecuatoriano.

Para hacerlo, se debe revisar a profundidad el tema del Retorno Marginal de Capital (MPK), mencionado en el Gráfico 4 y otras partes de este estudio, pero que se ha retrasado a propósito hasta este punto para analizarlo de una manera que explique la relación entre este mecanismo, la Dolarización y el Ecuador.

Comenzando por las definiciones, el retorno marginal de capital se refiere específicamente al aumento de la producción por la adición de un elemento más de capital. Pero para calcular los costos totales de producción, es necesario balancear este concepto con el retorno marginal del trabajo, denotado como MPL en este estudio. Obviamente, este será el retorno marginal de agregar 1 unidad adicional de trabajo, la cual puede ser una hora más de trabajo, o un trabajador adicional, dependiendo el cálculo que se esté realizando.

Siendo así, los costos relativamente más bajos de países subdesarrollados son los que atraen a las industrias extranjeras (transitivas) a instalarse en estos países, y hacer rendir las tecnologías desarrolladas. Esto a su vez crea una situación en donde el crecimiento económico estimado para el país subdesarrollado es mayor que el del país desarrollado que está en equilibrio, como se muestra debajo en el Gráfico 5:

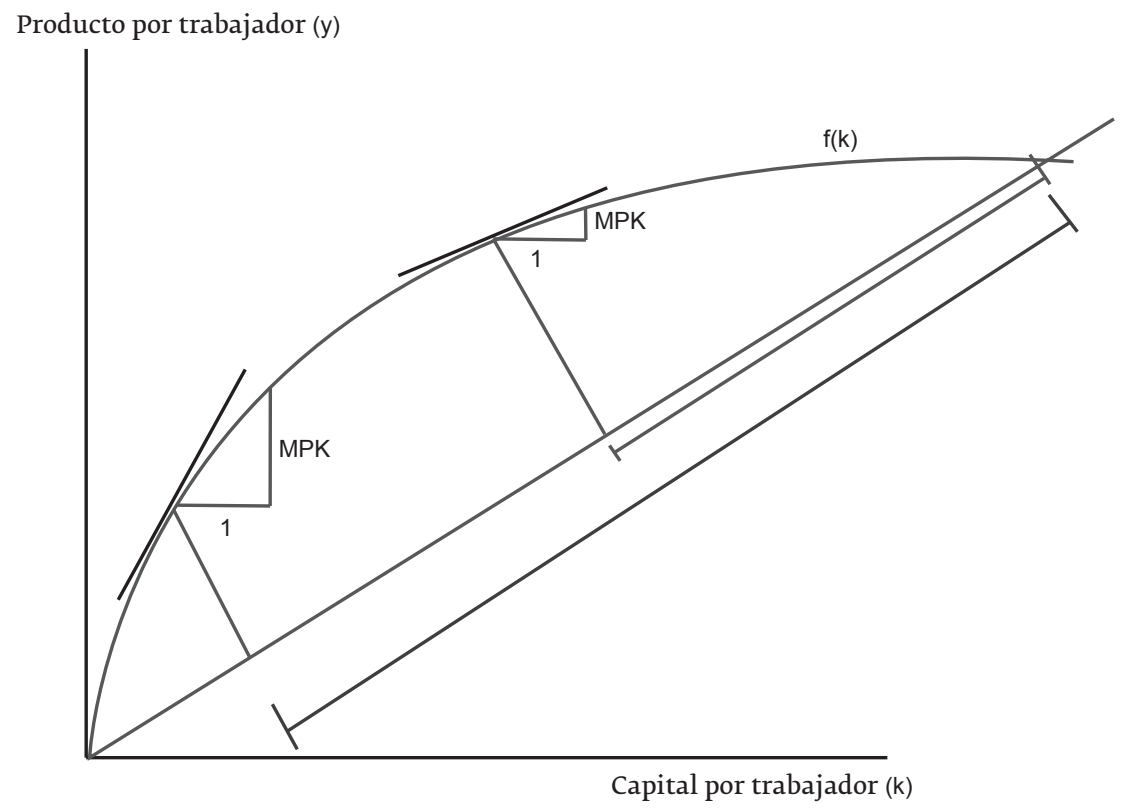

Figura 5. Curva de producción clásica

Lo que se puede observar arriba es algo que se había mostrado anteriormente: la curva MPK del país no desarrollado es más pronunciada (mayor pendiente) que la curva MPK del país desarrollado (curva azul), dan- do más campo de desarrollo, más oportunidad de crecimiento al país no desarrollado.

Lo que falta en este gráfico es una mayor explicación del por qué la curva está con mayor pendiente: básicamente esto se debe a que los 
costos totales del productor son más bajos en el país no desarrollado que en el desarrollado, lo cual, como se dijo antes, también depende del costo del trabajo (MPL). Por esto, es necesario recordar cual es la relación de MPL y MPK: Costos Totales del Productor:

$$
\mathrm{C}=\mathrm{wMPL}+\mathrm{rMPK}
$$

Esta clásica ecuación dice que los costos totales del productor serán iguales a lo pagado en salarios ( $w$, wages) multiplicado por el producto marginal del trabajo (MPL), sumado a los costos de arriendo del capital (interés, "r") (renta de inmuebles, financiamientos de máquinas, mantenimientos, etc), por el producto marginal del capital. Basados en esta ecuación, la relación MPK y MPL es:

$$
\frac{\mathrm{w}}{\mathrm{MPL}}=\frac{\mathrm{r}}{\mathrm{MPK}}
$$

Básicamente esta ecuación dice que el ratio de los salarios sobre el retorno del Trabajo (la productividad del trabajador), debe ser similar a los costos de renta sobre el retorno de capital (Weil, 2013). De la literatura económica estándar es posible definir la dinámica de los costos basados en la ecuación presentada. Normalmente un país es pobre (subdesarrollado) porque carece cosas, es decir, carece de capital. Esto causa que haya una gran demanda por este rubro, haciendo que cada unidad de capital que se agrega tenga gran productividad (gente queriendo usar el capital, más turnos de trabajo, más usos, etc). Esto hace que el capital produzca más, por lo cual el costo de adquirir este capital (r), decaiga rápidamente por unidad producida. Eso quiere decir que r/MPK será pequeño al ser $r$ fijo (intereses, costos fijos), pero MPK variable y, en este caso, creciente. Eso forza a que w/MPL sea bajo también.

En este punto también hay una gigantesca tangente que se va a evitar en este trabajo: el comparar la productividad de trabajadores de países subdesarrollados (Ecuador) con el de países desarrollados (Estados Unidos). Eso es larga literatura de otro tipo, como por ejemplo en Economía del Desarrollo o Economía Laboral, donde tratan temas como el ausentismo laboral, el estado de salud, la cultura, la educación, e incluso el tema de peligros morales en los trabajadores de diferentes tipos de países. Aquí no se va a considerar esos puntos, a propósito, dado que no es el tema principal del estudio. Aun así, tampoco se lo puede ignorar: los trabajadores de países subdesarrollados son menos productivos, así que la única forma en la que W/MPL sea equivalente a r/MPK es que w esté bajando estrepitosamente rápido, para compensar tanto la fuerte caída de r/MPK, así como que el MPL de los trabajadores siendo inferior al de los trabajadores de países desarrollados, incluso después de aumentar su productividad a través del incremento de capital.
El problema de Ecuador es que la dolarización no permite que los sueldos de los trabajadores (w) se ajuste de la manera arriba descrito, dado que no da campo a una manera en la cual esa w logre bajar a la velocidad necesaria para mantener este equilibrio, esto es, devaluando la moneda nacional, haciendo que los sueldos sean cada vez menores en términos reales.

Más allá de los problemas morales que esto presta, el tener salarios en moneda "dura" (no devaluable), hace que la ecuación w/MPK arroje un resultado más alto que el que debería en condiciones normales, haciendo que al mismo tiempo la productividad del trabajador ecuatoriano esté "sobrevalorada", no sólo en comparación a los países desarrollados sino que en comparación a otros países subdesarrollados que sí pueden permitirles a sus trabajadores reducir sus sueldos por devaluación.

Sin entrar en grandes cálculos matemáticos, es fácil ver que si w/MPK crece, entonces r/ MPL crece. Considerando que la renta de capital (r) es prácticamente fija puesto que las maquinas se venden en monedas duras (dólares, euros, otros), y que todos los suministros para mantenerlas trabajando son prácticamente globales, se puede considerar que la r no crecerá, por lo cual la única otra opción lógica para que r/MPK crezca es que se reduzca MPK, es decir, que caiga el producto marginal de capital. Entre más bajo sea el MPK, más alto será el resultado, e igualará el otro lado de la ecuación.

¿Hasta dónde debería bajar MPK en términos reales? Regresando a la estadística presentada en regresiones al inicio de este estudio (Tabla 2), se propone que el MPK en Ecuador va a bajar hasta igualarse con el de Estados Unidos, es decir, la hipótesis aquí presentada es que la dolarización crea un ancla alrededor de los salarios en Ecuador, que obliga a las otras variables de la ecuación de la producción a ajustarse a la misma, no reflejando la verdadera productividad del trabajador ecuatoriano, y al mismo tiempo causando que deba reducirse el producto marginal de capital para compensar. Es fácil ver por qué: si los trabajadores "cuestan" mucho, no van a haber 2 o 3 turnos para trabajar el capital, no van a haber horas extra, ni ninguna otra actividad que promueva el extensivo uso de ese capital.

Bajo esta óptica, lo que se propone es que la dolarización forza una pendiente de MPK sobre la economía ecuatoriana, que no es la que le corresponde en su condición de país en desarrollo (país pobre), lo cual causa una reducción [forzada] de su producción, y reduce su capacidad de crecimiento económico hasta igualar la del país "donante" de la moneda, en este caso Estados Unidos. Debajo se grafica este punto: 


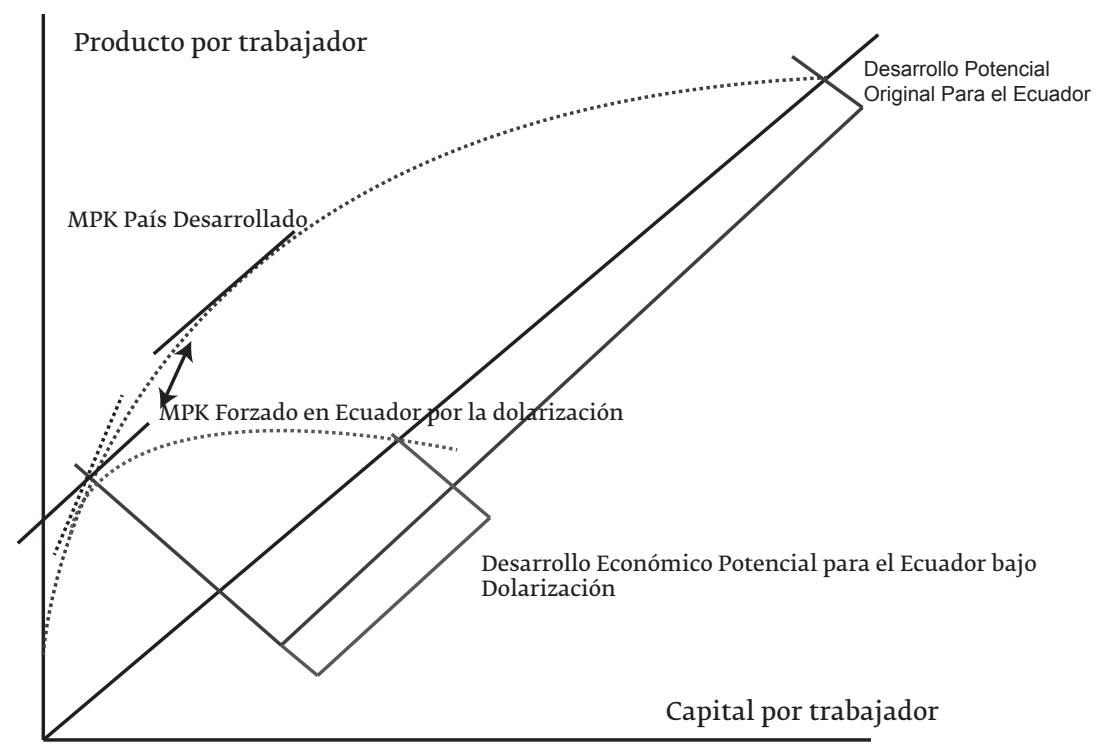

Figura 6. Desarrollo Económico Truncado del Ecuador debido a la Dolarización

La Figura 6 muestra la relación matemática presentada previamente, lo cual se puede observar en la sobreposición de la curva de MPK del país rico (USA) sobre la de donde debería ubicarse la de Ecuador. Esto causa varios efectos derivados, fácilmente observables. El primero lo muestra claramente el gráfico: la curva de producción de Ecuador se trunca, partiéndose y saliéndose de su cauce normal, cayendo rápidamente y formando un nuevo equilibrio. La razón por la cual la curva se trunca es que el punto de equilibrio de MPK es paralelo a la línea de población, ese es su punto más alto. Entonces, lo que sucede es que cuando ambas curvas se alinean, la curva de producción de Ecuador empieza a actuar como si hubiese alcanzado ese mismo punto de equilibrio, es decir, alcanza su ápex en relación a la curva de población+ depreciación, por lo cual cualquier cosa después de este punto es un retorno decreciente de producción, que es lo que refleja la caída de la curva mostrada.

El otro punto que muestra este gráfico es que al caer la curva, cae también el potencial de crecimiento económico de Ecuador. Hay que recordar que lo que se definió como crecimiento económico es un porcentaje de crecimiento del PIB, no una cantidad, por tanto no es que el gráfico (al menos directamente) esté limitando hasta donde puede crecer el PIB del Ecuador, lo que habla es de la velocidad con la que va a crecer, en este caso esa velocidad será mucho menor de lo que debería ser naturalmente, esto es, sin dolarización. A continuación, se muestra el por qué será igual a la de Estados Unidos:

Tabla 2 Transferencias tecnológicas y ganancias sociales de países desarrollados a sub-desarrollados

\begin{tabular}{lllllll}
\hline & \multicolumn{3}{c}{ ESTADOSUNDOS } & \multicolumn{2}{c}{ ECUADOR } \\
\hline & Cenerador & Inversionista & Sociedad & Cenerador & Inversionista & Sociedad \\
\hline IndustriaNoTransitiva & $\mathrm{Si} \uparrow$ & $\mathrm{Si} \uparrow$ & $\mathrm{Si} \uparrow$ & $\mathrm{Si} \uparrow$ & $\mathrm{S} \uparrow \uparrow$ & $\mathrm{S} \uparrow \uparrow$ \\
Clíster & $\mathrm{Si} \uparrow$ & $\mathrm{Si} \uparrow$ & Igual= & $\mathrm{Si} \uparrow$ & $\mathrm{S} \uparrow \uparrow$ & Igual \\
IndustriaTransitiva & $\mathrm{Si \uparrow}$ & Nada & Pierde $\downarrow$ & Nach & Nach & Pierde $\downarrow$ \\
\hline
\end{tabular}

Antes de comenzar a analizar la Tabla 4, es importante hacer el mismo ejercicio lógico que se hizo anteriormente: si Estados Unidos desarrolla tecnología, gana $2(3 \uparrow-1 \downarrow)$, por lo explicado anteriormente cuando se presentó la Tabla 3. En el caso de Ecuador, lo que se ha dicho es que las Industrias Transitivas no irán a Ecuador por los altos costos causados por la dolarización, sin embargo, los clústers existen- tes en Ecuador y las Industrias No Transitivas del país recibirán las tecnologías y las implementarán. Al no ir las industrias Transitivas a Ecuador, lo que causa es que ni los generadores de la tecnología ni los inversionistas pueden ganar si no hay la transferencia de tecnología. Sin embargo, el Ecuador sí termina perdiendo los puestos de trabajos que, con industrias transitivas mejoradas por la tecnología, 
ya no puede retener. Ejemplos tales como el descrito sobran en Ecuador desde la dolarización: la industria del calzado, de los textiles, automotriz, varios productos agrícolas, entre otras; es decir que sí pierde. El resultado final es que la cuenta para Ecuador, después de la transferencia tecnológica, es la misma que la de Estados Unidos: gana 2 netos ( $3 \uparrow-1 \downarrow)$.

El único resultado lógico de esta situación es que Estados Unidos y Ecuador deben tener el mismo crecimiento económico en el largo plazo. Esto implica a su vez que la dolarización impone un límite a cuánto puede crecer el Ecuador, convirtiéndose así en la "manga alta" de la camisa de fuerza económica que es Ecuador. La siguiente sección tratará sobre la otra manga faltante: la "manga baja".

\section{Manga Alta: La Dolarización y la} Transferencia Tecnológica

Hasta la sección anterior se mostró el límite superior de la dolarización mediante la prevención de que industrias transitivas de países desarrollados se instalen en Ecuador. En esta sección lo que se va a intentar demostrar es que la dolarización garantiza que las tecnologías que van hacia los otros dos tipos de industria, no transitivas y clústers, lleguen y se implementen en Ecuador con celeridad para crear crecimiento a través de esos sectores; y con particular énfasis en las industrias No Transitivas debido a que, como se ha referido anteriormente, los clústers son cadenas de valor global por lo cual para funcionar dependen mucho menos de las situaciones individuales de los países donde estén ubicados.

Para entender por qué la Dolarización cierra esta "tenaza" del crecimiento económico creando una situación de avance casi forzado, hay que entender la coyuntura del Ecuador en este momento: al día de escribir este acápite, el Presidente de la República le acaba de quitar las funciones a la vicepresidenta, acusada de corrupción. Peor aún, es la segunda persona en el cargo: en Enero de este mismo año fue destituido el vicepresidente anterior por el mismo cargo. Es decir, nuevamente el Ecuador está inmerso en los vaivenes políticos y la inestabilidad característica del pasado pre-dolarización. Sin embargo cabe la pregunta, ¿cuál es el efecto de todo esto sobre los mercados financieros y el funcionamiento de la economía en el país? La respuesta es ninguno, gracias a la dolarización. Y de este hecho se puede desprender que la dolarización implica que estos mercados funcionan desconectados del resto del país, casi como si fueran un outsourcing.

Siendo así, la pregunta es por qué la dolarización facilitaría que se dé la transferencia tecnológica hacia el Ecuador, versus por ejemplo otros países de la región. Primeramente, se recuerda que la transferencia tecnológica de la que se habla es hacia las industrias No Transitivas, puesto que las Transitivas definitivamente no vendrán al país como se expuso anteriormente. Además, pese a que los clústers pueden ser financiados internamente en un país, nuevamente estos son animales de otras especies, más internacionales, por lo cual podrían potencialmente autofinanciarse sin depender en el sistema financiero del país.

¿Cómo logra la dolarización esta adquisición y permeación tecnológica en Ecuador? Lo hace creando la base sobre la cual se asienta un sistema financiero que funciona como el de una economía desarrollada, sin el Ecuador ser una. ¿Qué significa esto? Para empezar, como se comentó arriba, lo "blinda" de los choques sociales y políticos del país. Esto a su vez implica que hay confianza en el sistema financiero, confianza que se traduce en crédito, palabra que viene del latín "creer”. Este crecimiento se ve reflejado en el extraordinario aumento de los depósitos en el sistema financiero nacional desde la dolarización. En el gráfico que se presenta debajo se observa como en el periodo comprendido entre el 2007 y el 2015, los depósitos en el sistema financiero ecuatoriano subieron de US\$900o millones a US\$21000 millones, es decir más del doble. El contraste es incluso más profundo si se lo toma desde el momento de la dolarización a la fecha: en Diciembre de 1999 los depósitos en el sistema financiero eran de $\$ 2300$ millones, mientras que al cierre de este año serán de \$27.0oo millones, un aumento de una magnitud superior a 10 veces, una cifra que se mantendría incluso luego de descontar la inflación de estos años (Banco Central del Ecuador, 2018). 
Depósitos Cuentas Corrientes + Cuasidinero del Panorama Financiero

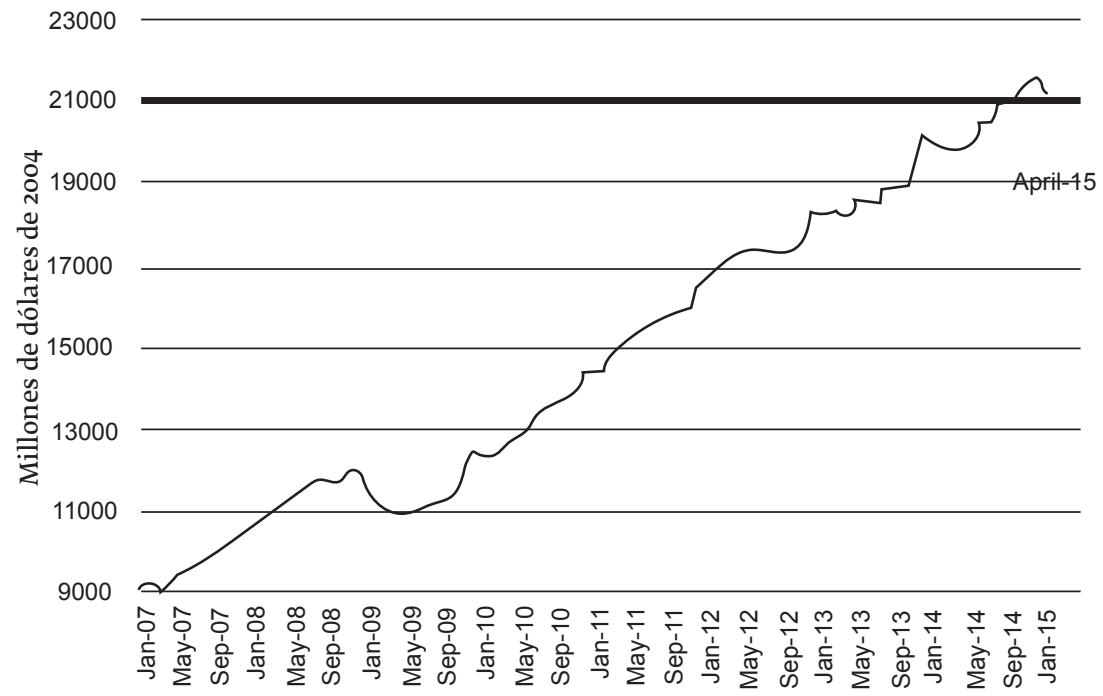

Figura 7. Evolución de los depósitos en Ecuador Adaptado de Economía en Jeep (Gonzalez, 2015)

¿Cuál es el impacto de mayores depósitos en el sistema nacional? La teoría monetaria más básica enseña que el aumento de capital hace que los intermediarios financieros busquen colocar esos valores, y para lograrlo bajan las tasas de interés de los préstamos y créditos a niveles mucho más competitivos que los que le correspondería a un país como el Ecuador. Esto a su vez hace que se vuelvan rentables proyectos que antes tenían retornos muy bajos para serlo (Rao, 2014), especialmente aquellos que se promovían en las épocas de mega-devaluaciones por encima del $20 \%$ anual, que era muy común en la etapa pre-dolarización.

Proyectos rentables y líneas de crédito disponibles atraen a los emprendedores, pero también a los inversionistas, lo cual se refleja en un saludable crecimiento del mercado de valores en Ecuador en la etapa post-dolarización, con un respetable $3,2 \%$ anual, pasando de US\$2711 millones en el 2000 a US\$6617 millones para el 2017 (Jiménez, 2018), esto es casi se ha triplicado su tamaño en este periodo. El atraer inversionistas de este tipo es importante para la implementación tecnológica dado que los mismos permiten horizontes más largos para los proyectos, los cuales son necesarios para lograr ver los resultados de la nueva tecnología, especialmente si se deben realizar instalaciones para equipos, adquirir y adecuar terrenos, comprar algún insumo especializado, obtener permisos, etc. Para resumir se presente el siguiente gráfico que muestra como la Dolarización fomenta la implementación tecnológica en Ecuador:

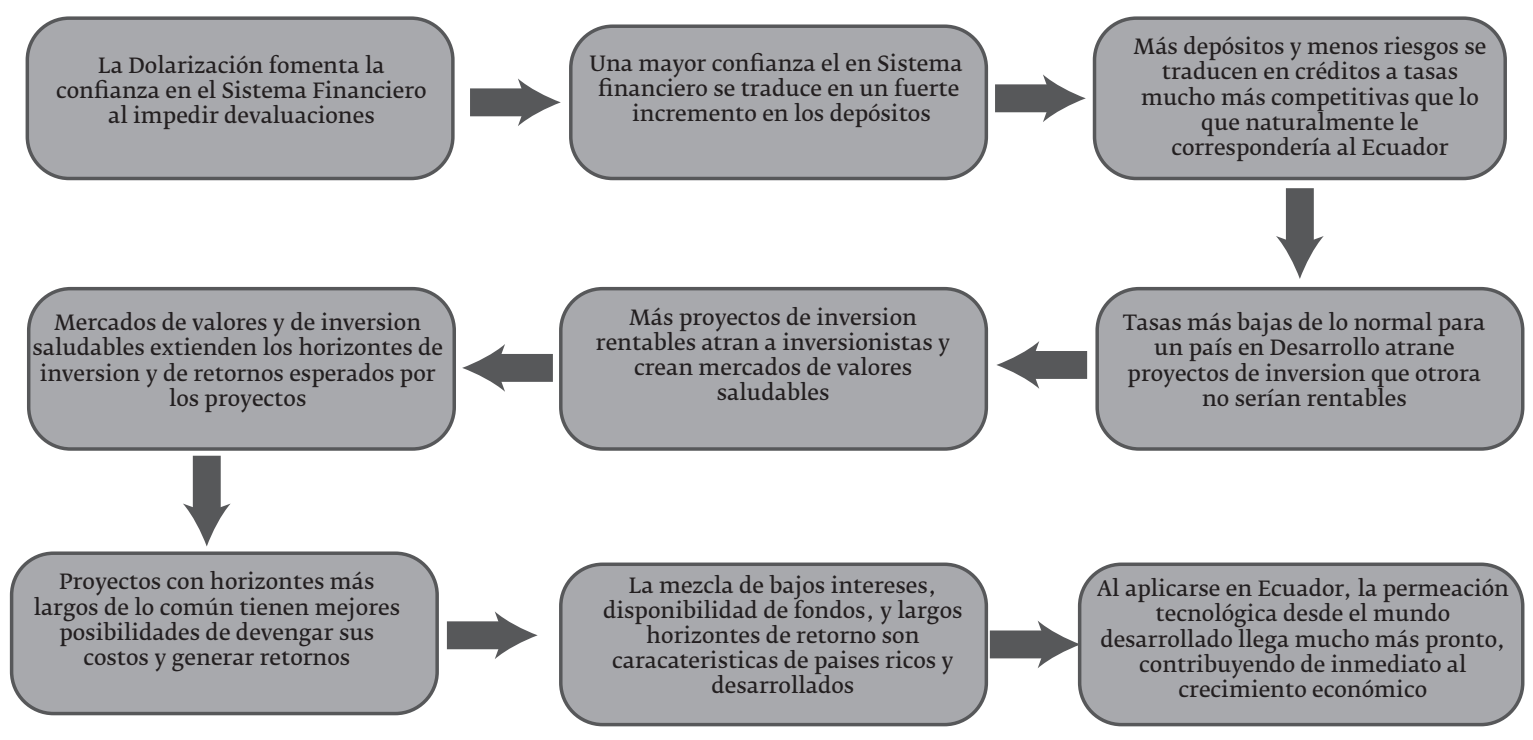

Figura 8. La dolarización y la permiación tecnológica 
Para finalizar, hay que entender los dos elementos que hacen que este crecimiento se vea forzado a darse, contrario al acápite anterior donde el crecimiento se truncaba. Aquí, la dolarización crea las condiciones necesarias para que los proyectos dirigidos a mejorar las industrias locales que sean competitivas se puedan implementar con una probabilidad razonablemente alta de éxito, tal y como lo explica en anterior gráfico. Además, crea un alto costo de oportunidad en no hacerlo: si aun habiendo las condiciones adecuadas para realizar un proyecto el sector privado decidiese no hacerlo por situaciones ajenas al mercado (es decir, que en vez de rentabilidad se estén fijando en temas políticos con el gobierno de turno, temas culturales o sociales como el racismo, xenofobia, etc), puede venir otra persona o inversionista y tomar la posta del proyecto, quedándose con toda la ganancia. En un caso así la sociedad no pierde, sólo el empresario o grupo empresarial que decidió no realizarlo.

Por ende, esta "manga baja" de la dolarización es la que "empuja” al Ecuador hacia el crecimiento económico lo quiera o no, al darle una situación de mercado financiero con garantías comparables al de un país desarrollado, sin que el Ecuador esté ni remotamente cercano a tal realidad.

\section{Discusión}

El primer gran punto del presente estudio es el de presentar una dinámica coherente y basada sobre bases teóricas sólidas respecto a por qué el Dolarizar ralentiza el crecimiento económico de un país. En Ecuador este no ha sido un tema investigado en su justa medida, pero por suerte, Ecuador no es el único país dolarizado del continente: otro país dolarizado en la mis- ma época (pero con una mucho mejor planeación), fue El Salvador, donde se ha detectado el mismo efecto de la Dolarización sobre el crecimiento económico pero sin dar ninguna luz respecto al por qué. (Rodriguez \& Dombrow, 2016) Consideramos que el presente resuelve estas dudas, o, por lo menos, da una dirección hacia dónde encontrar la solución de esta interrogante, usando las teorías aquí planteadas.

Segundo, la contribución del presente ensayo se basa sobre lo presentado anteriormente respecto a que la inflación no es la camisa de fuerza de la Dolarización (Calle, 2018), presentando una alternativa viable que satisface el cálculo matemático que predice que al fijar una variable (la tasa cambiaria), es decir, convertirla en constante; alguna otra variable debía fijarse para compensar ese cambio. No fue la inflación, pero la evidencia, tanto de Ecuador como de El Salvador, parece apuntar a que sí es el crecimiento económico.

Tercero, se vuelve importante discutir que el tema de crecimiento económico y convergencia, siendo esta última la capacidad de los países en desarrollo de alcanzar el nivel de actividad económica y bienestar de los países desarrollados. Para entender este punto es necesario revisar nuevamente la literatura respecto a las dinámicas de convergencia económica de Solow. Propuesto en 1959 en conjunto entre Robert Solow y Trevor Swan, el modelo intenta explicar el crecimiento económico a largo plazo mediante el uso de variables como la acumulación de capital, el trabajo (labor), crecimiento poblacional, etc. (Parker, 2017)

Este tópico es extenso, superando ampliamente el presente trabajo, por lo cual el enfoque se centrará en lo que el modelo de Solow pronostica en el siguiente gráfico:

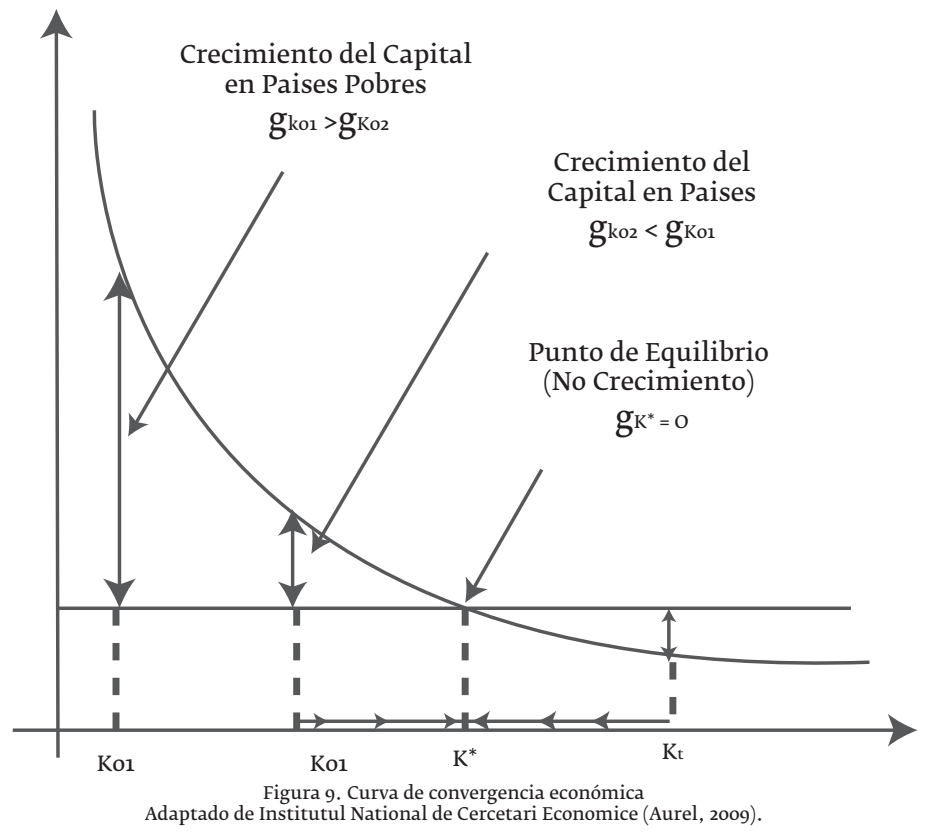


Lo que el gráfico anterior muestra son las diferencias de tasas de crecimiento entre países pobres y países ricos, lo que es lo mismo, entre países desarrollados y países no desarrollados. Lo que se debe destacar de este modelo es que predice que el crecimiento de los países pobres no solo será más grande que el de los países desarrollados, sino que este se dará de una manera más acelerada, lo cual se deriva de la forma geométrica de la curva (la pendiente de los países subdesarrollados es más pronunciada).

La razón principal por la cual los países subdesarrollados crecen más rápido es porque su Producto Marginal de Capital (MPK) es mayor cuando están fuera de equilibrio, tal y como se mencionó provisionalmente en el Gráfico 4. Este trabajo, contrario a lo que se pudiera interpretar, no descarta que se dé esta posibilidad, sino que plantea que se lo hará si el país dolarizado logra crecer al nivel (o cerca del nivel) del PIB potencial del país ancla, en este caso Estados Unidos. Cabe recordar que el PIB Potencial es el punto de la triple coincidencia macroeconómica donde está el nivel de inflación más bajo con el nivel de empleo más alto y el nivel de crecimiento más elevado posible.

Insistimos en decir que es muy difícil que un país en desarrollo, con todas las limitantes sociales y educacionales que tiene, pueda superar a Estados Unidos en el manejo económico para lograr estar en o cerca de su PIB Potencial, sin embargo, esa es la vía disponible para convergir económicamente y brindarle un bienestar más alto a su población, por ende está más que ser una meta técnica para un país dolarizado debiera ser un mandato moral de su gobierno.

\section{Conclusiones}

La primera conclusión que debería derivarse de este trabajo es la novedosa interpretación tanto de los modelos de crecimiento endógeno por tecnología de Romer, como el de crecimiento económico de Solow. La introducción de la Dolarización como un elemento disruptor, el cual sumado a la definición de país rico o pobre basado en la capacidad de creación de tecnología, crea una situación en la cual se demuestra exactamente lo opuesto a lo que propuso Romer: el crecimiento tecnológico puede ser exógeno (como lo demuestra el Ecuador siendo un país con demostrada nula innovación tecnológica); y el crecimiento económico en sí mismo puede pasar de endógeno a condicionado o forzado, como se trató arriba en las conclusiones generales. Hasta donde el autor conoce, esta es la primera vez que se ha usado el modelo de Romer y el de Solow de esta manera.

La segunda conclusión sería dejar pendiente el hacer un seguimiento al crecimiento del PIB real de Ecuador y el de Estados Unidos, y tratar de esta manera de corroborar si las ideas aquí expuestas efectivamente se realizan, o si se debe hacer algún ajuste. Este seguimiento debería ir más allá de sólo ver las cifras, sino que debería también incluir un seguimiento econométrico respecto a los crecimientos, alertando de cualquier ajuste o cualquier nuevo elemento que se deba incluir.

Una tercera conclusión es el investigar y contrastar las realidades del mercado laboral ecuatoriano, utilizando herramientas formales de economía del trabajo las cuales no se han aplicado aquí, sobre todo por falta de espacio. Las leyes y diferentes aristas de ambos mercados laborales hace que sea un trabajo de investigación propio, más allá de lo que se puede cubrir aquí, sin embargo sería importante revisarlo para tener una idea de qué tan cerca o tan lejos de la verdad está la idea de que el MPK de ambos países es similar.

Una cuarta conclusión académica que se deriva de este trabajo corresponde al señoreaje. Se ha propuesto en este trabajo que el señoreaje es la Fuerza de la camisa de fuerza, pero no se especificó la dimensión de esta fuerza. Es más, no se ha propuesto cómo hacer este cálculo. Considerando que en el 2019 Ecuador cumple 20 años dolarizado, sería interesante saber si este es un valor constante o variable, $y$ en lo segundo, si es lineal o geométrico. Por ejemplo, ¿sería sólo el valor equivalente del punto óptimo de señoreaje del 2018, o sería equivalente al punto óptimo del señoreaje del año 2000 , multiplicado por 20? ¿O peor aún, sería compuesto de la forma (señoreaje 2000)20?

Este cálculo podría servir para hacer algo que fascina pregonar a los políticos ecuatorianos pero que ya se ha demostrado que es imposible: desdolarizar. Insistiendo en que esta no es una opción técnica, no deja de ser interesante la pregunta de cuánto sería el valor real de hacerlo. Mejor aún, con un sistema establecido, se podría actualizar este valor de manera constante, para monitoreo económico.

La conclusión final de este ensayo es que todo plan económico del Ecuador debe comenzar por los estimados de crecimiento económico de la economía de Estados Unidos, tanto nominales como potenciales. Esta será la meta de crecimiento del Ecuador para cada año, con ciertos grados de libertad dentro de un rango razonable. El crecimiento de la economía americana debería servir como un piso mínimo para todo gobierno de turno: lo mínimo que el Ecuador debería crecer es la misma magnitud, cualquier cantidad menor implica que el gobierno está ejerciendo alguna injerencia negativa que no deja fluir el mecanismo económico aquí presentado de la forma natural en que debería darse.

La convergencia a la que deben apuntar todas las gestiones de los gobiernos de países en desarrollo (o, directamente, subdesarrollados), es posible que todavía llegue bajo este esquema 
cuando se nota que el techo no es el crecimiento nominal sino el crecimiento potencial del país ancla (en el caso de Ecuador ese país es Estados Unidos). Pensar que se puedan hacer las cosas de una forma en que la economía ecuatoriana crezca de manera más ágil que la americana (es decir, con menos trabas que le permitan realizar su potencial total); es un tanto utópico, pero no imposible, cuando se considera que Ecuador es un país mucho más pequeño, con menos población, más homogéneo, y sin gastos ni goteras en la economía (leakages) como son los compromisos en el exterior que tiene Estados Unidos (como las guerras, las transferencias de ayuda externa, etc). El pensamiento final debe ser que es difícil, pero con disciplina y constancia, es posible de lograrse.

\section{Referencias}

Aurel, I. , (2009). Real Economic Convergence, Bucarest, Rumania: InstitutulNationaldeCercetariEconomice(INCE).

Barossi-Filho, M., Gonçalves, R. \& Martins, E. (2004). The Empirics of The Solow Growth Model: LongTerm Evidence, Sao Paulo: University of Sao Paulo. Bernanke, B. \& Gtirkaynak, R. (2001). Is Growth Exogenous? Washington DC: NBER Macroeconomics Annual.

Calle, E. (2018). Convergencia Inflacionaria: La Gran Mentira de la Dolarización, Guayaquil: Revista Espirales.

Calle, E. (2019). Crecimiento Economico: la verdadera camisa defuerzadela Dolarizacion, Guayaquil: Revista Espirales .

Carroll, C. (2017). The Romer (1986) Model of Growth, Baltimore: Johns Hopkins University.

Castillo, R. \& Rodriguez, L., 2009. La dolarización de El Salvador: ¿una contribución negativa al crecimiento económico?, San Salvador: comercio exterior, vol. 59, núm. 11, noviembre de 2009 .

Cely, N. \& Calle, E. J. (2019). Examining the Foreign Trade dynamics of Ecuador under Dollarization using Ricardian Theories, Delray Beach, FL: Journal of Asia Pacific Studies (2019) Volume 5 No 3, 688-715.

Diewert, E. et al. (2011). Returns to scale: concept, estimation and analysis of Japan's turbulent 1964-88 economy, Toronto: Canadian Journal of Economics.

El Universo (2011). La Bahía de Guayaquil, en el 'top cinco' mundial de la piratería. El Universo, 19 Junio.

Gonzalez, M. (2015). Los Depósitos de la Economía se Siguen Reduciendo en Términos Reales. [Online]

Available at: http://economiaenjeep.blogspot.com/2015/o4/ los-depositos-de-la-economia-se-siguen.h tm

Hartley, M. \& Walker, C. (2012). The Culture shock of India's Call Centers. [Online]
Available at: https://www.forbes.com/sites/morganhartley/2012/12/16/the-culture-shock-of-indias-call-centers/\#322c72d972f 5

Ibarra, D., Moreno-Brid, J. C. \& Perez, E. (2001). La dolarización: antecedentes y perspectivas para la economía mexicana, Caracas: Revista Nueva Sociedad.

Isaac, A. (2017). Macro Notes, Washington, Dc: American University.

Jones, C. I. (1995). R \& D-Based Models of Economic Growth, Chicago: Journal of Political Economy.

Mankiw, G. (2007). Macroeconomics, 6 th edition. 6th ed. New York: Worth Publishers.

Mixon, W. (2007). The Solow Growth Model: An Excel-Based Primer, Londrés: The Journal of Economic Education.

Nudelsman, S. (2017). Los regímenes cambiarios en América Latina, Buenos Aires: Ensayos de Economia No 51.

Parker, J. (2017). Solow Growth Model: Exposition, Portland, Oregon: Reed College. Policonomics, (2017). Robert Solow. [Online] Available at: https://policonomics.com/es/robert-solow/ [Accessed 9 July 2019]. Rao, A., (2014). Innovation and Interest Rates. [Online] Available at: https://slate.com/business/2014/o2/ in novation-and-interest-rates. h t m l Redacción BBC (2018). Nobel de Economía: quién es Paul Romer, ganador del premio y por qué provocó que Chile llamara "inmoral" al Banco Mundial. [Online]

Available at: https://www.bbc.com/mundo/noticias-america-latina-45784664 $\begin{array}{llll}\text { [Accessed } & 9 & \text { Julio } & \text { 2019]. } \\ \text { Robb, A., (2018). The Costs \& Bene- }\end{array}$ fits of New Technology. [Online] Available at: https://study.com/academy/lesson/ the-costs-benefits-of-new-technology.htm

Rodriguez, M. \& Dombrow, J., (2016). ¿Por qué la dolarización frenó el crecimiento en El Salvador?, San Salvador: El Faro Académico.

Sachs, J. \& Larrain, F., (1999). Why Dollarization Is More Straitjacket Than Salvation, Washington DC: Foreign Policy No. 116 (Autumn, 1999), pp. 80-92 (13 pages).

Sollow, R., (1994). Perspectives on Growth Theory, New York: Journal of Economic Perspectives

Thurow, L. \& Taylor, L., (1966). The Interaction Between the Actual and the Potential Rates of Growth, Boston: The Review of Economics and Statistics Vol. 48, No. 4 (Nov., 1966), pp. 351-36o.

Whelan, K., (2014). Endogenous Technological Change: The RomerModel, Dublin, Irlanda: University College Dublin.

Williams, N., (2017). The Solow Model and Convergence, Madison, Wisconsin: University of Wisconsin - Madison.

WIPO, (2017). World Intellectual Property Indicators 2017, Ginebra: WIPO.

WriteOpinions, (2004). Opinions on Endogenous growth theory. [Online] Available at: http://www.writeopinions.com/endogenous-growth-theory 